\title{
Efeito do dinitrato de isossorbida sobre a hiperalgesia corneana induzida por lipopolissacarídeo
}

\author{
Isosorbide dinitrate effect on corneal hyperalgesia \\ Induced by lipopolysaccharide
}

Jayter Silva Paula', Mariana Bellini Oliveira', Fernando Chahud², Carolina Maria Modulo', Eduardo Melani Rocha' Fernando Queiróz Cunha ${ }^{3}$.

\section{Resumo}

Objetivo: Avaliar o efeito do dinitrato de isossorbida (DNI) sobre a hiperalgesia corneana e a infiltração de neutrófilos em um modelo experimental de ceratite superficial induzida pelo lipopolissacarídeo (LPS). Métodos: A hiperalgesia foi estudada através da indução de uma ceratite inflamatória em ratos $(n=60)$, pela exposição da córnea ao LPS $($ LPS +). Após a exposição, os olhos foram tratados durante quatro dias, com as soluções tópicas: DNI (200 mg, $65 \mathrm{mg}$ e $20 \mathrm{mg}$ ), prednisolona $1 \%$ (PRED) ou Veículo. Olhos controles foram expostos apenas à solução salina (LPS -). Para avaliar a dor ocular, contouse o número de piscadas em 40 segundos, após instilação de uma gota de capsaicina $0,01 \mathrm{mM}$. A análise histopatológica foi realizada para avaliação da infiltração neutrofílica. Resultados: Foram observadas áreas esbranquiçadas no estroma corneano dos olhos com a ceratite induzida pelo LPS, entre os dias 3 e 15 dias. Esses olhos (LPS +) apresentaram um número significativamente maior de piscadas que os LPS - $(\mathrm{P}=0,019)$ à estimulação química. O tratamento tópico com o DNI reduziu o número de piscadas observadas $(\mathrm{P}=0,010)$. Da mesma forma, olhos expostos ao LPS e tratados com DNI demonstraram redução significativa na infiltração neutrofílica $(P=0,0031)$. Conclusão: Baixas doses de DNI reduziram a hiperalgesia corneana e a infiltração neutrofílica nesse modelo de ceratite. Dessa forma, doadores de óxido nítrico, como o DNI, poderão ser úteis no futuro ao tratamento clínico de diversas condições dolorosas da superfície ocular.

Descritores: Dinitrato de isossorbida/uso terapêutico; Hiperalgesia/induzido quimicamente; Lipopolissacarídeos/ efeitos adversos

\section{AbStract}

Purpose: To analyze effects of isosorbide dinitrate (DNI) on corneal hyperalgesia and neutrophil infiltration in an experimental model of superficial keratitis induced by lipopolysaccharide (LPS). Methods: Hyperalgesia was studied through the induction of inflammatory keratitis in rats $(n=60)$, by corneal exposure to LPS. Following exposure, the inflamed eye was treated for four days, with one of the following solutions: topical DNI (200 $\mu \mathrm{g}, 65 \mu \mathrm{g}$ and $20 \mu \mathrm{g})$, prednisolone 1\% (PRED), and vehicle. Saline-exposed eyes (LPS -) underwent the same protocol. To evaluate ocular pain, the number of blinks in 40 seconds was counted, after one drop of $0.01 \mu \mathrm{M}$ capsaicin. Histopathological analysis was performed with evaluation of neutrophil infiltration. Results: White clouding areas were observed in the corneal stroma of eyes with LPS-induced keratitis, between day 3 and day 15. Eyes exposed to LPS had a significantly higher number of blinks than LPS - $(P=0.019)$. Topical treatment of LPS-induced keratitis eyes with DNI reduced capsaicin-induced blinks $(P=0.010)$. Similarly, eyes exposed to LPS and treated with DNI also displayed reduced neutrophil infiltration $(P=0.0031)$. Conclusion: Low doses of topical NO donors, like DNI, reduce corneal hyperalgesia and neutrophil infiltration in this keratitis model. NO donors may be useful in the clinical treatment of painful conditions associated with surgical procedures of the ocular surface.

Keywords: Isosorbide dinitrate/therapeutic use; Hyperalgesia/chemically induced; Lipopolysaccharides/adverse effects

'Departamento de Oftalmologia, Otorrinolaringologia e Cirurgia de Cabeça e Pescoço, Faculdade de Medicina de Ribeirão Preto, Universidade de São Paulo - USP -Ribeirão Preto (SP), Brasil.

${ }^{2}$ Departamento de Patologia, Faculdade de Medicina de Ribeirão Preto, Universidade de São Paulo - USP -Ribeirão Preto (SP), Brasil.

${ }^{3}$ Departamento de Farmacologia, Faculdade de Medicina de Ribeirão Preto, Universidade de São Paulo - USP -Ribeirão Preto (SP), Brasil.

Faculdade de Medicina de Ribeirão Preto, Universidade de São Paulo - USP -Ribeirão Preto (SP), Brasil.

Os autores declaram inexistir conflitos de interesse

Fontes de auxílios: FAPESP, CNPq, FAEPA.

Recebido para publicação em 20/10/2010 - Aceito para publicação em 19/1/2011 


\section{INTRODUÇÃO}

$\mathbf{O}$ óxido nítrico (NO) é uma molécula gasosa altamente difusível envolvida em muitas funções fisiológicas $^{(1)}$, podendo também apresentar efeitos pró e anti-inflamatórios. Ele modula a migração de neutrófilos através de mecanismos de redução na expressão de moléculas de adesão ${ }^{(2,3)}$.

Há evidências do envolvimento do $\mathrm{NO}$ em processos nociceptivos $^{(4)}$. O uso de precursores de NO pode promover a redução da dor de origem central e periférica. De fato, a administração intraplantar ${ }^{(5,6)}$ ou subcutâneo ${ }^{(7)}$ de um precursor de NO (L-arginina) reduziu a hiperalgesia evocada pela carragenina, em testes de pressão plantar. Esta substância também foi capaz de diminuir alguns quadros de dor crônica em pacientes ${ }^{(8)}$.

Na córnea, condições associadas à inflamação descontrolada são geralmente dolorosas e podem resultar em opacificação estromal e perda da visão. Colírios com efeitos analgésico ou anestésico podem retardar o processo de cicatrização, o que pode levar a sérias complicações secundárias da córnea, como defeito epitelial recorrente e cicatrizes ${ }^{(9-11)}$.

A fim de investigar o mecanismo subjacente ao desencadeamento de inflamações da córnea, diversos modelos experimentais têm sido propostos, incluindo os que envolvem agentes pró-inflamatórios, como o lipopolissacarídeo da endotoxina bacteriana (LPS) ${ }^{(12,13)}$. O LPS está envolvido na produção sequencial de diversos mediadores inflamatórios, incluindo prostaglandinas, leucotrienos e aminas simpatomiméticas ${ }^{(14-16)}$, e podem mediar o aumento da hiperalgesia e infiltração leucocitária ${ }^{(17)}$. Sendo o NO um mediador identificado em estudos anteriores de inflamação corneana ${ }^{(18)}$, torna-se interessante verificar o possível efeito de doadores de NO, como o dinitrato de isossorbida (DNI), em um modelo experimental de ceratite inflamatória induzida pelo LPS.

\section{Métodos}

Todos os procedimentos experimentais foram aprovados pelo Comitê de Ética em Experimentação Animal da Faculdade de Medicina de Ribeirão Preto - Universidade de São Paulo. Os animais foram tratados em conformidade com as orientações estabelecidas pela Association for Research in Vision and Ophthalmology (ARVO).

\section{Animais}

Ratos Wistar de oito semanas de idade foram alojados em gaiolas com ração e água ad libitum, mantidas em ciclos claro/escuro de $12 \mathrm{~h}$.

\section{Preparação das soluções}

A capsaicina (Sigma Chemical Co., St. Louis, MO) foi dissolvida em etanol e Tween-20 e, em seguida, diluída em solução tampão fosfato (PBS) (concentração final: $0,003 \%$ EtOH, $0,003 \%$ de Tween-20), sendo que o dinitrato de isossorbida e a prednisolona (Sigma Chemical Co., St. Louis, MO) foram diluídos apenas em PBS. Todos os medicamentos foram aplicados na córnea em um volume de $5 \mathrm{uL}$ de solução. As soluções apresentavam $\mathrm{pH}$ de 7,0 e ficaram protegidas da luz e calor.

\section{Indução de inflamação corneana}

O estudo foi conduzido de acordo com o seguinte protocolo: todos os animais foram anestesiados com éter etílico, por via inalatória, previamente aos procedimentos de abrasão corneana. Com uma agulha estéril 26G, procedeu-se uma raspagem contínua do epitélio corneano de ambos os olhos (de limbo a limbo e horizontalmente - Figura $1 \mathrm{~A})$ como descrito anteriormente ${ }^{(13)}$. Com base nesses protocolos e ensaios pilotos, verificou-se que $50 \mu \mathrm{g}$ de LPS (endotoxina pseudomonas aeruginosa serótipo B4 - pureza de 99,7\%, Sigma Chemical Co., St. Louis, MO) diluída em salina estéril apirogênica (grupos LPS +) apresentou melhores resultados quando aplicado topicamente por 30 segundos nas córneas escarificadas (dados não apresentados). Como controle (grupos LPS -), córneas escarificadas receberam apenas solução salina estéril apirogênica. Todos os olhos foram novamente raspados e expostos à solução de LPS tópica ( $5 \mu \mathrm{g}$, durante 20 segundos) ou apenas salina estéril apirogênica, 72 horas após o primeiro procedimento, a fim de potencializar o processo inflamatório.

\section{Procedimentos experimentais}

Logo após a primeira abrasão e exposição ao LPS, os olhos foram tratados a cada duas horas, cinco vezes ao dia, com uma das soluções de teste a seguir: PBS (veículo-VEH), DNI nas concentrações de 200 $\mu \mathrm{g} / 5 \mu \mathrm{l}$ (DNI1), $65 \mu \mathrm{g} / 55 \mu \mathrm{l}$ (DNI2) ou $20 \mu \mathrm{g} / 55 \mu \mathrm{l}$ (DNI3), e prednisolona $1 \%$ tópico (PRED). O mesmo protocolo de tratamento foi utilizado nos animais não expostos ao LPS (grupos LPS -). Seis ratos foram testados por grupo experimental para cada fármaco e dosagem, e o período de tratamento foi de quatro dias.

Em todos os grupos, os ratos foram examinados diariamente utilizando lâmpada de fenda e as córneas foram documentadas através de fotografias. Em testes preliminares, a avaliação comportamental da dor foi estabelecida com base em modificações de estudos anteriores $^{(19)}$. A avaliação objetiva do comportamento de 


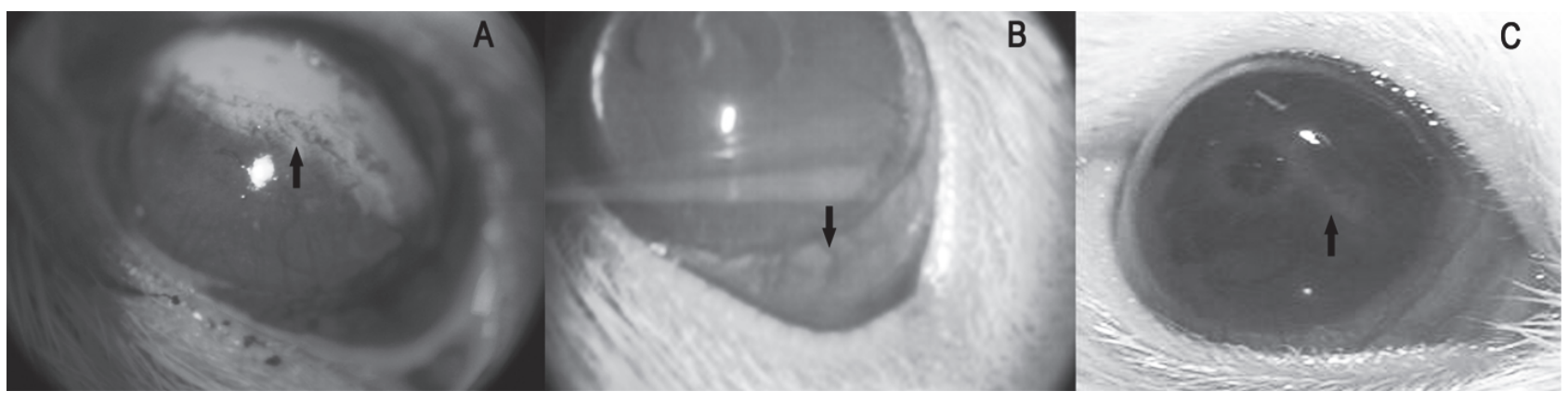

Figura 1: Fotografias de olhos de ratos Wistar durante a indução de ceratite inflamatória; A) Marcação positiva com fluoresceína nas áreas da abrasão epitelial linear (seta preta); B) Observar hiperemia conjuntival moderada no dia 3 (seta preta); C) Nubéculas na área paracentral do estroma corneano observadas após sete dias (seta preta)

dor ocular, dado pelo desafio químico com capsaicina suficiente para iniciar uma resposta nociceptiva, foi determinada no dia 4 pela contagem do número de piscadas contadas em 40 segundos, após a instilação de uma gota com $0,01 \mu \mathrm{M}$ de capsaicina. Inicialmente, os animais foram aclimatados em um ambiente tranquilo e calmo por, pelo menos, uma hora antes da análise comportamental. Na sequência, os animais foram adaptados em recipientes de acrílico por pelo menos 5 minutos. Cada olho foi testado uma única vez, para se evitar outras alterações potencialmente induzidas pela capsaicina, como a perda de sensibilidade periférica.

Após os testes nociceptivos, elegeu-se para análise histológica os grupos LPS -, LPS+ (VEH), LPS+ tratado com DNI na concentração que produziu os melhores resultados em termos de controle da hiperalgesia (DNI2) e LPS+/PRED. Quatro animais de cada um desses grupos foram anestesiados com éter e sacrificados no dia 4, por deslocamento cervical, para avaliação histopatológica.

\section{Histologia}

Após o sacrifício, os olhos foram enucleados, incluídos em OCT 4585 (Tissue-Tek, Miles, Elkhart, IN) e congelados em nitrogênio líquido. Procedeu-se então preparação histológica de rotina para coloração em hematoxilina e eosina (HE), através de cortes de $10 \mu \mathrm{m}$ em criostato e montagem em lâminas gelatinizadas. Todas as lâminas foram montadas de forma a se orientar os cortes transversais paralelamente à área de abrasão. Os neutrófilos foram contados de limbo a limbo em cortes histológicos seriados de toda córnea de cada animal, por um patologista experiente. A média do número de neutrófilos em cada córnea foi utilizada para análise estatística.

\section{Análise estatística}

Os resultados estão apresentados em gráficos, com média ( \pm erro padrão da média;EPM). As diferenças no número de piscadas e nos parâmetros histológicos, entre os grupos, foram avaliadas com os testes não paramétricos de Kruskal-Wallis ou Mann-Whitney U (bicaudal), sendo valores de $\mathrm{P}<0,05$ considerados estatisticamente significativos.

\section{Resultados}

\section{Achados anatômicos}

A análise biomicroscópica dos animais, incluindo a dos testes preliminares, revelou áreas brancas de opacificação no estroma corneano dos olhos expostos ao LPS, entre os dias 3 e 15 (Figura 1). Após o dia 15, não se observou evidência de inflamação e todos os animais recuperaram o aspecto normal da córnea, sem sinais de neovascularização corneana. Não foi identificado também nenhum sinal de uveíte, em todos os olhos estudados.

Os parâmetros clínicos utilizados para análise biomicroscópica foram a hiperemia ocular relacionada à congestão de vasos da conjuntiva e episcleral, opacificação da córnea, neovascularização, edema epitelial, e estromal, e pontos hemorrágicos. Os olhos do grupo LPS- mostraram pouca ou nenhuma reação inflamatória. Em contraste, os olhos expostos ao LPS (LPS+) apresentaram resposta inflamatória mais intensa. No dia 3, observou-se hiperemia conjuntival moderada e neovascularização corneana periférica fina (Figura 1B). Nubéculas na área paracentral do estroma corneano foram observadas a partir do dia 4 , após a segunda exposição ao LPS (Figura 1C).

\section{Comportamento nociceptivo}

A estimulação química com capsaicina nos olhos expostos ao LPS e tratados com o veículo, apresentaram um número significativamente maior de piscadas do que aqueles do grupo LPS - (7,4 $\pm 1,3$ versus $3,0 \pm 0,8$ pisca- 
das, $\mathrm{P}=0,019)$, dado este indicativo de maior hiperalgesia no grupo expostos ao LPS. O tratamento tópico da ceratite inflamatória induzida por LPS com $65 \mathrm{ug} / 5 \mu \mathrm{L}$ DNI (DNI2) produziu redução significativa no número de piscadas desencadeadas pela capsaicina $(2,2 \pm 0,4$ piscadas, $\mathrm{P}=0,010)$, de forma semelhante ao observado no grupo PRED $(2,7 \pm 0,4$ piscadas, $\mathrm{P}=0,011)$ (Figura 2). Olhos tratados com outras concentrações de DNI apresentaram menores reduções no número de piscadas (DNI1: 5,2 $\pm 0,9$ piscadas - $\mathrm{P}=0,2448$ e DNI3: $2,2 \pm 0,6$ piscadas - $\mathrm{P}=0,0157$ ). Curiosamente, os olhos não expostos ao LPS, mas tratados com qualquer concentração de DNI (LPS -/DNI) apresentaram um número significativamente maior na média de piscadas em relação ao grupo LPS -/VEH $(\mathrm{P}=0,029)$.

\section{Achados histológicos}

Uma vez que o grupo DNI2 exibiu os melhores resultados na avaliação nociceptiva, este grupo, além dos controles (LPS-) e dos tratados com PRED, foi eleito para processamento histológico. Foi observado um pronunciado infiltrado celular no estroma corneano anterior nos grupos expostos ao LPS, dado primordialmente por neutrófilos, diferentemente do apresentado em córneas não expostas ao LPS. Essas células se localizavam principalmente próximas aos vasos limbares no grupo LPS $+(70,4 \pm 3,8$ versus $6,3 \pm 0,5$ células, teste Kruskal-Wallis - $\mathrm{P}<0,0001)$. Os grupos DNI 2 e PRED apresentaram redução significativa na infiltração de neutrófilos $(49.6 \pm 4.9$ células $-P=0.0031$ e $27.5 \pm 13.2$ células - $P=0.0306$, respectivamente) (Figura 3).

\section{Discussão}

Quadros inflamatórios da córnea, também conhecidos como ceratites, podem ser desencadeados por hipóxia, trauma, agentes microbianos e agressão autoimune. Normalmente, a ceratite causa dor e desconforto e pode levar à perda visual permanente por cicatrização e, ocasionalmente, perfuração da córnea ${ }^{(20)}$.

A ceratite também pode se desenvolver na ausência de bactérias, sob a forma de infiltrados periféricos. Estes infiltrados estéreis, frequentemente associados com o uso de lentes de contato, podem ser condições dolorosas que resultam, menos frequentemente, em deficiência visual(21). Embora a etiologia de infiltrados estéreis não tenha sido completamente definida, a noção de envolvimento de partículas bacterianas parece razoável, dado que alguns produtos bacterianos podem ser liberados após lise de bactérias presentes na lágrima e que foram observados infiltrados neutrofílicos importan-

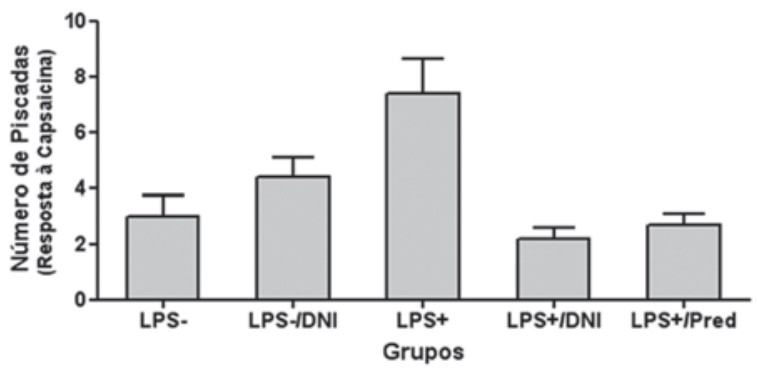

Figura 2. Distribuição do número de piscadas em resposta a uma solução 0,01 mM de capsaicina aplicada a córneas inflamadas (LPS +) ou não (LPS -). DNI, dinitrato de isossorbida (65ig/5il); LPS, lipopolissacarídeo; PRED, prednisolona $1 \%$. Os resultados representam a média $\pm E P M$.

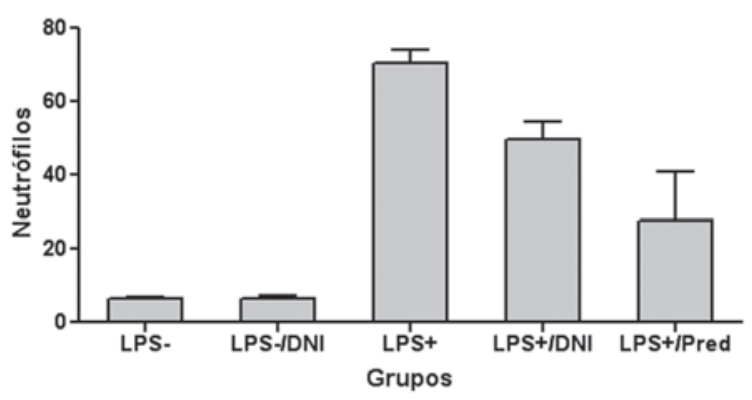

Figura 3: Distribuição do número médio de neutrófilos contados por corte histológico das córneas estudadas. LPS -/DNI e LPS -: animais não expostos ao LPS, tratados e não tratados com DNI, respectivamente; $L P S+/ D N I$ e $L P S+$ : animais expostos ao LPS, tratados e não tratados com DNI $(65 \mu \mathrm{g} / 5 \mu \mathrm{l})$, respectivamente. LPS +/PRED: animais expostos ao LPS e tratados prednisolona 1\%. Os resultados representam a média $\pm E P M$

tes em biópsias de pacientes com esse tipo de ceratite ${ }^{(21)}$. O presente estudo apresenta um modelo experimental de ceratite, com metodologia adequada para avaliar a hiperalgesia corneana, dada pela utilização de um estímulo químico conhecido. Tal modelo se mostrou útil para testar novas drogas com potencial terapêutico para processos patológicos da superfície ocular. As alterações induzidas pelo LPS foram confirmadas pelo desenvolvimento de nubéculas no estroma de córneas expostas ao LPS, exclusivamente. Essa ceratite induzida por LPS parece ser resultado direto do recrutamento de neutrófilos para os locais estimulados no estroma corneano. A inflamação observada ficou confinada à conjuntiva, limbo e córnea, uma vez que, nenhum sinal de uveíte foi observado.

Consistente com os resultados apresentados, estu-

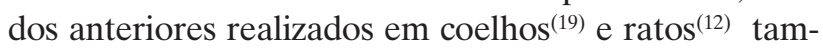
bém demonstraram que a aplicação de LPS induziu a migração de neutrófilos para o estroma da córnea. Também de acordo com os presentes achados, estudos anteriores sugerem que o recrutamento de neutrófilos para áre- 
as centrais da córnea após a exposição ao LPS se deu a partir de vasos sanguíneos do limbo e da conjuntiva, apesar do filme lacrimal pode ser fonte de neutrófilos ${ }^{(22)}$.

O dinitrato de isossorbida (DNI) é conhecido por seu efeito vasodilatador, ocasionado pela liberação local de NO nos processos inflamatórios. O NO também está envolvido na modulação da dor, tanto a nível periférico quanto central. A administração periférica de doadores de NO em animais de experimentação ou em humanos produziu resultados discrepantes.

A injeção intraplantar de 3-morfolinosidnonimina (SIN-1) pode induzir hiperalgesia no teste de pressão de pata $^{(23)}$, enquanto que nitroprussiato de sódio ${ }^{(5)}$, SIN-1 (em concentrações menores: 50-100 $\mu \mathrm{g})^{(14)}$ ou S-nitroso$\mathrm{N}$-acetil-DL-penicilamina (SNAP) $(50-200 \mu \mathrm{g})^{(16)}$ reduz a hiperalgesia mecânica induzida pela prostaglandinaE2. Apesar deste efeito controverso sobre o controle nociceptivo periférico, a liberação intracelular de baixas concentrações de NO está relacionado à redução da dor devido à ativação da guanilato-ciclase ${ }^{(24)}$.

Com o intuito de aumentar a nossa compreensão da hiperalgesia corneana e o efeito do NO no controle do status nociceptivo periférico, avaliou-se o efeito de doses baixas de DNI (como um doador de NO) no controle da hiperalgesia corneana. O DNI produziu uma redução substancial (até $70 \%$ ) no comportamento nociceptivo dos animais provocado por uma dose padronizada de capsaicina, com resultados comparáveis aos do corticóide usado (64\%). Os olhos tratados com DNI e PRED também apresentaram diminuição do número de neutrófilos no estroma corneano, porém este efeito foi menos pronunciado nos animais tratados com DNI (30\% versus $60 \%$ para PRED, aproximadamente). Outros estudos têm mostrado a ação antiangiogênico do NO em queimaduras alcalinas graves da córnea, no entanto sem demonstração de análises comportamentais de dor ${ }^{(18)}$. Este efeito antiangiogênico do NO não pode ser avaliado neste estudo, uma vez que todos os animais apresentaram regressão total da neovascularização periférica.

Levando em consideração os dados mencionados, pode-se dizer que o efeito anti-hiperalgésico do DNI tópico pode depender de um aumento direto nos níveis de GMPc nos neurônios sensitivos primários ou de um efeito indireto relacionado à redução da migração de neutrófilos. Seguindo este raciocínio, há evidências na literatura que o aumento do GMPc nos neurônios sensitivos reduz a hiperalgesia ${ }^{(25)}$. Além disso, estudos anteriores $^{(26)}$ demonstraram que neutrófilos que migraram para o sítio inflamatório são uma fonte importante de mediadores, como as prostaglandinas, que sensibilizam os nociceptores. Assim, especula-se se baixas doses de doa- dores de NO poderiam atuar direta e/ou indiretamente, ativando a guanilato ciclase solúvel e/ou inibindo a migração de neutrófilos respectivamente, com redução da hiperalgesia periférica.

A real utilidade clínica de um tratamento com doadores tópicos de $\mathrm{NO}$ em quadros de ceratite séptica e asséptica, bem como após procedimentos cirúrgicos, deve ser explorada como uma alternativa para o controle da dor ocular, especialmente em situações em que o uso de esteróides tópicos pode ser prejudicial. Os doadores de NO também podem apresentar vantagens sobre os esteróides, uma vez que não apresentam os efeitos oculares adversos destes, como por exemplo, a catarata e o glaucoma. Neste contexto o efeito microbicida do $\mathrm{NO}^{(27)}$ seria uma propriedade adicional útil, primordialmente no tratamento de doenças infecciosas dolorosas da superfície ocular.

\section{Conclusões}

Em resumo, nossos resultados revelaram que o modelo de ceratite induzida por LPS é adequado para o estudo da hiperalgesia relacionada com a inflamação de estruturas da superfície ocular. Além disso, os dados sugerem que o DNI pode reduzir a hiperalgesia induzida quimicamente na córnea, com diminuição da infiltração neutrofílica. Experimentos futuros serão necessários para investigar a relação entre esses efeitos e os mecanismos fisiológicos envolvidos.

\section{REFERÊNCIAS}

1. Schuman EM, Madison DV. Nitric oxide and synaptic function. Annu Rev Neurosci. 1994;17:153-83.

2. Moncada S, Palmer RM, Higgs EA. Nitric oxide: physiology, pathophysiology, and pharmacology. Pharmacol Rev. 1991;43(2):109-42. Review.

3. Dal Secco D, Moreira AP, Freitas A, Silva JS, Rossi MA, Ferreira SH, Cunha FQ. Nitric oxide inhibits neutrophil migration by a mechanism dependent on ICAM-1: role of soluble guanylate cyclase. Nitric Oxide. 2006;15(1):77-86.

4. Meller ST, Gebhart GF. Nitric oxide (NO) and nociceptive processing in the spinal cord. Pain. 1993;52(2):127-36. Comment in: Pain. 1993;55(3):401-3.

5. Duarte ID, Lorenzetti BB, Ferreira SH. Peripheral analgesia and activation of the nitric oxide-cyclic GMP pathway. Eur J Pharmacol. 1990;186(2-3):289-93.

6. Nakamura A, Fujita M, Shiomi H. Involvement of endogenous nitric oxide in the mechanism of bradykinin-induced peripheral hyperalgesia. Br J Pharmacol. 1996;117(3):407-12.

7. Kawabata A, Nishimura Y, Takagi H. L-leucyl-L-arginine, naltrindole and D-arginine block antinociception elicited by L-arginine in mice with carrageenin-induced hyperalgesia. Br J Pharmacol. 1992;107(4):1096-101. 
8. Harima A, Shimizu H, Takagi H. Analgesic effect of L-arginine in patients with persistent pain. Eur J Neuropsychopharmacol. 1991;1(4):529-33.

9. Willis WE, Laibson PR. Corneal complications of topical anesthetic abuse. Can J Ophthalmol. 1970;5(3):239-43.

10. Burstein NL, Klyce SD. Electrophysiologic and morphologic effects of ophthalmic preparations on rabbit cornea ephitelium. Invest Ophthalmol Vis Sci. 1977;16(10):899-911.

11. Rocha G, Brunette I, Le Francois M. Severe toxic keratopathy secondary to topical anesthesic abuse. Can J Ophthalmol. 1995;30(4):198-202.

12. Khatri S, Lass JH, Heinzel FP, Petroll WM, Gomez J, Diaconu $E$, et al. Regulation of endotoxin-induced keratitis by PECAM-1, MIP-2, and toll-like receptor 4. Invest Ophthalmol Vis Sci. 2002;43(7):2278-84.

13. Schultz CL, Morck DW, McKay SG, Olson ME, Buret A. Lipopolysaccharide induced acute red eye and corneal ulcers. Exp Eye Res. 1997;64(1):3-9.

14. Ferreira SH, Duarte ID, Lorenzetti BB. The molecular mechanism of action of peripheral morphine analgesia: stimulation of the cGMP system via nitric oxide release. Eur J Pharmacol. 1991;201(1):121-2.

15. Cunha FQ, Poole S, Lorenzetti BB, Ferreira SH. The pivotal role of tumor necrosis factor alpha in the development of inflammatory hyperalgesia. Br J Pharmacol. 1992;107(3):660-4.

16. Cunha FQ, Teixeira MM, Ferreira SH. Pharmacological modulation of secondary mediator systems-cyclic AMP and cyclic GMP - on inflammatory hyperalgesia. Br J Pharmacol. 1999;127(3):671-8.

17. Sachs D, Cunha FQ, Poole S, Ferreira SH. Tumour necrosis factor-alpha, interleukin-1beta and interleukin-8 induce persistent mechanical nociceptor hypersensitivity. Pain. 2002;96(1-2):89-97.

18. Sennlaub F, Courtois Y, Goureau O. Nitric oxide synthase-II is expressed in severe corneal alkali burns and inhibits neovascularization. Invest Ophtalmol Vis Sci. 1999;40(12):2773-9.

19. Wenk HN, Honda CN. Silver nitrate cauterization: characterization of a new model of corneal inflammation and hyperalgesia in rat. Pain. 2003;105(3):393-401
20. Whitcher JP, Srinivasan M, Upadhyay MP. Corneal blindness: a global perspective. Bull World Health Organ. 2001;79(3):214-21.

21. Holden BA, Reddy MK, Sankaridurg PR, Buddi R, Sharma S, Willcox MD, et al. Contact lens-induced peripheral ulcers with extended wear of disposable hydrogel lenses: histopathologic observations on the nature and type of corneal infiltrate. Cornea. 1999;18(5):538-43.

22. Chusid MJ, Davis SD. Polymorphonuclear leukocyte kinetics in experimentally induced keratitis. Arch Ophthalmol. 1985;103(2):270-4.

23. Aley KO, McCarter G, Levine JD. Nitric oxide signalling in pain and nociceptor sensitization in the rat. J Neurosci. 1998;18(17):7008-14.

24. Prado WA, Schiavon VF, Cunha FQ. Dual effect of local application of nitric oxide donors in a model of incision pain in rats. Eur J Pharmacol. 2002;441(1-2):57-65.

25. Sachs D, Cunha FQ, Ferreira SH. Peripheral analgesic blockade of hypernociception: activation of arginine/NO/cGMP/ protein kinase G/ATP-sensitive $\mathrm{K}+$ channel pathway. Proc Natl Acad Sci U S A. 2004;101(10):3680-5.

26. Cunha TM, Verri WA Jr, Schivo IR, Napimoga MH, Parada CA, Poole S, et al. Crucial role of neutrophils in the development of mechanical inflammatory hypernociception. J Leukoc Biol. 2008;83(4):824-32.

27. Colasanti M, Gradoni L, Mattu M, Persichini T, Salvati L, Venturini G, Ascenzi P. Molecular bases for the anti-parasitic effect of NO (Review). Int J Mol Med. 2002;9(2):131-4. Review.

Autor de Correspondência:

Prof. Dr. Jayter Silva Paula

Departamento de Oftalmologia, Otorrinolaringologia

e Cirurgia de Cabeça e Pescoço

Faculdade de Medicina de Ribeirão Preto - USP

Avenida Bandeirantes, $\mathrm{n}^{\circ} 3900$.

CEP 14049-900 - Ribeirão Preto, SP

Telefone / Fax: 55(16) 3602-3602-2523/3602-2499

E-mail: jspaula@fmrp.usp.br 